1 Universidade Federal de Alfenas (Unifal) - Varginha (MG), Brasil.

Orcid: https://orcid org/0000-0003-08924841 anamarciarodrigues@gmail. com

2 Universidade de São Paulo (USP), Escola de Enfermagem - Ribeirão Preto (SP), Brasil. Orcid: https://orcid. org/0000-0001-94129515

sergiovalverdemarques@ hotmail.com

3 Universidade de São Paulo (USP), Escola de Enfermagem - Ribeirão Preto (SP), Brasil. Orcid: https://orcid org/0000-0002-00333740

carloshdfl@gmail.com

4 Universidade Federal de Alfenas (Unifal) - Varginha (MG), Brasil. Orcid: https://orcid org/0000-0003-03584980

deborajplima@gmail.com

5 Universidade de São Paulo (USP), Escola de Enfermagem - Ribeirão Preto (SP), Brasil. Orcid: https://orcid org/0000-0003-23645787

avrmlccr@eerp.usp.br

\section{Fatores associados à prática de atividade física entre trabalhadores brasileiros}

\author{
Factors associated to the practice of physical activity by Brazilian \\ workers
}

Ana Marcia Rodrigues da Silva', Sérgio Valverde Marques dos Santos², Carlos Henrique de Freitas Lima ${ }^{3}$, Debora Juliene Pereira Lima ${ }^{4}$, Maria Lucia do Carmo Cruz Robazzi5

DOI: 10.1590/0103-1104201811913

RESUMO Avaliaram-se os fatores associados à prática de atividade física de trabalhadores brasileiros. Trata-se de estudo descritivo, analítico, transversal, quantitativo, realizado com 82.019.207 trabalhadores por meio da Pesquisa Nacional por Amostra de Domicílios de 2015. Para verificar a associação da prática de atividade física dos trabalhadores, estimou-se a Odds Ratio (OR) com $\mathrm{p}<0,05$ por meio de modelo de regressão logística com Intervalo de Confiança (IC) de $95 \%$. Verificou-se que somente $31,7 \%$ dos trabalhadores praticavam atividade física. A possibilidade de praticar diminuiu com o aumento da idade, principalmente para aqueles do sexo masculino entre 46 e 55 anos (OR 0,626, IC 0,565-0,693), bem como para as trabalhadoras que possuíam filhos menores de 14 anos (OR 0,569, IC 0,474-0,682) e com elevada carga horária laboral. Do trabalho informal para o formal, ocorreu aumento da prática em 17,9\%. Tanto a elevação da escolaridade como a salarial aumentaram essa chance em, respectivamente, 5,719 para aqueles com quinze anos ou mais de estudo e em 2,365 para aqueles que auferem renda entre doze e quinze salários mínimos. Por fim, os militares possuíam maior chance de praticar atividade física (OR 5,586, IC 3,572-8,736).

PALAVRAS-CHAVE Saúde do trabalhador. Atividade física. Saúde pública. Trabalho.

ABSTRACT The factors associated with the practice of physical activity among Brazilian workers were evaluated. It is a descriptive, analytical, cross-sectional, quantitative study conducted with 82,019,207 workers by means of the National Survey by Household Sample of 2015. So to verify the association of physical activity practice among workers, Odds Ratio (OR) was estimated at $p<0.05$ by means of a logistic regression model with a 95\% Confidence Interval (CI). It was noted that only $31.7 \%$ of the workers practiced physical activity. The practicing probability decreased with age increasing, especially for males between 46 and 55 years old (OR 0.626, CI 0.565-0.693), as well as for female workers with children under 14 years old (OR 0.569, IC 0.474-0.682) facing high working hours. The practice of formal workers is 17.9\% higher than among informal workers. Also, the increase in schooling as in salaries raised the chance by, respectively, 5.719 for those with 15 years or more of study and by 2.365 for those between 12 and 15 minimum salaries of income. Finally, the military personnel enjoyed a greater chance of practicing physical activity (OR 5.586, IC 3.572-8.736).

KEY WORDS Occupational health. Exercise. Public health. Work. 


\section{Introdução}

Com as mudanças no perfil epidemiológico mundial, as causas de morte de caráter infectocontagioso cederam espaço para um elevado índice de mortalidade por Doenças Crônicas Não Transmissíveis (DCNT), totalizando 38 milhões de óbitos em 2012². No Brasil, segundo os dados da Global Burden of Disease Study, as DCNT foram responsáveis por $75 \%$ das mortes em $2015^{2}$. Entre os fatores que as desencadeiam, está a inatividade física ${ }^{3}$. Neste contexto, a prática regular de exercícios torna-se um instrumento importante para a promoção da saúde e a prevenção das DCNT ${ }^{4,5}$.

Todo movimento corporal realizado pelos músculos esqueléticos que seja capaz de gerar gasto de energia acima do estado de repouso com o objetivo de aperfeiçoar ou manter as condições de aptidão física é considerado atividade física. Quando tal atividade é competitiva e institucionalizada, pode ser caracterizada como esporte, devendo atender a regras e a aspectos técnicos ${ }^{6}$. Entre adultos, a recomendação global para a prática de atividade física é de, pelo menos, 150 minutos semanais de atividade leve ou moderada ou, no mínimo, 75 minutos quando é intensa ${ }^{5}$.

A inatividade física tornou-se um fator de risco para a mortalidade em nível mundial. Estimativas preveem que $31 \%$ da população mundial não seguem as recomendações para tal prática ${ }^{8}$. Nesse sentido, a inatividade tem sido responsável por mais de cinco milhões de mortes no mundo todos os anos ${ }^{4}$.

Essa inatividade é frequentemente justificada em razão das transformações no mundo do trabalho, pelas longas jornadas laborais, pelas transformações tecnológicas e pelo lazer hipocinético ${ }^{9}$. É fato que o mundo do trabalho sofreu transformações estruturais, principalmente, com os avanços tecnológicos que, embora tenham produzido benefícios para a população, têm causado a redução da qualidade de vida e da saúde dos trabalhadores. Tal fato é decorrente das modificações do mundo globalizado ${ }^{10}$.
Importante de se destacar com essas mudanças é que o trabalho tende a ocupar muito tempo do trabalhador, podendo diminuir seu convívio social, lazer e cuidados com a saúde, o que o torna vulnerável à exaustão, ao adoecimento ocupacional e à inatividade física".

A Organização Mundial da Saúde (OMS) estabeleceu a meta global para redução de $10 \%$ nos níveis de inatividade física da população em geral, tendo em vista o enfrentamento das DCNT. Por isso, o Brasil adotou metas semelhantes contra essas enfermidades para o período 2011-2022, o que torna imprescindível o monitoramento dos níveis desse tipo de atividade da população ${ }^{2,5,12}$.

Frente ao exposto e devido aos problemas que a inatividade pode causar aos indivíduos e, em especial, aos trabalhadores que possuem longas jornadas de trabalho, objetivou-se neste estudo analisar os fatores associados à prática de atividade física de trabalhadores brasileiros. Tal análise se justifica porque os resultados podem auxiliar e monitorar os níveis dessa prática entre a população em estudo. Isso se faz necessário para um melhor acompanhamento das políticas de promoção à saúde. Além disso, esta investigação agrega conhecimento em virtude de sua abrangência nacional, diferentemente do caráter local e focado da maioria dos estudos relacionados a esta temática.

\section{Material e métodos}

Trata-se de estudo descritivo-analítico, transversal e de abordagem quantitativa, desenvolvido por meio dos dados da Pesquisa Nacional por Amostra de Domicílios (PNAD) do ano de 2015. A PNAD é uma pesquisa domiciliar realizada pelo Instituto Brasileiro de Geografia e Estatística (IBGE) em todo território nacional, a cada ano. Caracteriza-se, ainda, por pesquisa com amostragem probabilística que possui plano amostral estratificado e conglomerado com um, dois ou três estágios, conforme o estrato ${ }^{13}$.

O ano de 2015 foi escolhido em razão da realização, nesse ano, de pesquisa suplementar 
à PNAD sobre práticas de atividade física ${ }^{\mathbf{1 4}}$. Além disso, é o mais recente de divulgação da pesquisa, uma vez que a PNAD do tipo contínua mais recente não possui informações referentes a esse tema. Assim, este estudo foi realizado conforme a disponibilidade das variáveis no banco de dados da PNAD de 2015.

Com o intuito de verificar a prática de atividade física de indivíduos que trabalharam na semana de realização da pesquisa, 20 a 26.9.2015, considerou-se na análise frequência maior ou igual a vinte minutos por dia, duas vezes ou mais por semana, no tempo livre do trabalhador, conforme definido pela PNAD $2015^{14}$. Como tempo livre, considerou-se o horário que não incluía os afazeres domésticos, a educação física na escola e o percurso de ida e volta para o trabalho ou escola.

Para excluir os menores sujeitos a trabalho infantil, foram utilizados na análise os trabalhadores com idade maior ou igual a 16 anos. Dessa forma, a população total analisada foi de 82.019.207 indivíduos.

Inicialmente, a estatística descritiva foi aplicada com o objetivo de se conhecer o perfil dos trabalhadores que praticavam exercícios físicos versus os que não praticavam. Posteriormente, estimou-se um modelo de regressão logística a fim de verificar os fatores associados às razões de chances (Odds Ratio) dos praticantes de atividade física ao nível de significância de $5 \%$.

Neste estudo, a variável de interesse definida foi a prática de atividade física de trabalhadores. As variáveis explicativas foram o sexo, cor ou raça, idade, escolaridade, estado civil, tipo de família, condição de domicílio, região do domicílio, horas trabalhadas, salário, formalidade no trabalho e posição na ocupação.

Os dados foram estimados e analisados por meio do Programa Stata, versão 11. Por default, o Stata toma a primeira categoria de cada variável como categoria de referência para a análise do comportamento das variáveis explicativas em relação à variável explicada. Por esse motivo, não foram estimadas as Odds Ratio para essas categorias.

Com relação aos aspectos éticos, as informações secundárias extraídas da PNAD são de domínio público. Dessa maneira, não foi necessária a autorização para utilização dos dados nem a apreciação de algum Comitê de Ética em Pesquisa, conforme a Resolução Nacional no $466 / 2012^{15}$.

\section{Resultados}

Dos 82.019.207 trabalhadores considerados na análise, $25.564 .948(31,8 \%)$ praticavam atividade física. Destes, a maior parte praticava de quatro a sete vezes por semana, de 40 minutos a uma hora por dia. Com relação às razões que os levaram a essa prática, $38,0 \%$ foram motivados pelo objetivo de melhorar sua qualidade de vida ou bem-estar (tabela 1).

Tabela 1. Prática de atividade física entre os trabalhadores, conforme o tempo praticado e o motivo. Brasil, 2015 ( $n=$ 25.564.948)

\begin{tabular}{lrr}
\hline & Frequência absoluta & Frequência relativa (\%) \\
\hline Quantas vezes praticava atividade física por semana & & \\
\hline Duas & 7001329 & 27,4 \\
Três & 8945368 & 35,0 \\
Quatro a sete & 9618251 & 37,6 \\
\hline Tempo que destinava à prática de atividade física & & 15,1 \\
\hline Mais de 20 a 40 minutos no dia & 3864563 & 45,5 \\
\hline
\end{tabular}


Tabela 1. (cont.)

\begin{tabular}{lrr}
\hline Mais de 1 hora no dia & 9943221 & 38,9 \\
Não se enquadrava & 116898 & 0,5 \\
\hline Por que motivo praticou atividade física & & 12,1 \\
\hline Indicação médica & 3087940 & 25,2 \\
Melhorar ou manter o desempenho físico & 6452681 & 38,0 \\
Melhorar a qualidade de vida ou o bem-estar & 9723036 & 5,3 \\
Gosta de competir & 1365041 & 16,9 \\
Relaxar ou se divertir & 4309088 & 1,6 \\
Socializar encontrando com amigos ou fazer novas amizades & 401997 & 0,9 \\
Outro & 225165 & \\
\hline
\end{tabular}

Fonte: Elaboração com base nos dados da PNAD de 2015.

Em relação aos motivos dos que não praticavam atividades físicas, a maioria alegou a falta de tempo (50,3\%), 33,3\% afirmaram não gostar ou não querer e 10,0\% alegaram problemas financeiros. Os 6,3\% dos restantes tiveram suas justificativas distribuídas entre problemas de saúde, falta de acesso às atividades, falta de companhia ou outra causa não especificada.
Os dados mostram que o percentual de mulheres trabalhadoras que praticava atividade física era ligeiramente superior ao de homens. Os brancos eram menos sedentários e o mesmo foi observado nos indivíduos entre 16 e 25 anos e entre aqueles com maior escolaridade (tabela 2).

Tabela 2. Características pessoais dos trabalhadores em relação à pratica de atividade física. Brasil, 2015 ( $n=82.019 .207)$

\begin{tabular}{|c|c|c|c|c|c|c|}
\hline Variável & $\begin{array}{r}\text { Praticou atividade } \\
\text { física }\end{array}$ & $\begin{array}{r}\text { Não praticou } \\
\text { atividade física }\end{array}$ & Odds Ratio & Valor $\mathrm{p}$ & \multicolumn{2}{|c|}{$\begin{array}{r}\text { Intervalo de } \\
\text { Confiança }\end{array}$} \\
\hline & $n=25.564 .948$ & $n=56.454 .259$ & & & & \\
\hline \multicolumn{7}{|l|}{ Sexo } \\
\hline Feminino & $32,0 \%$ & $68,0 \%$ & & & & \\
\hline Masculino & $30,5 \%$ & $69,5 \%$ & 0,976 & 0,424 & 0,920 & 1,036 \\
\hline \multicolumn{7}{|l|}{ Cor ou Raça } \\
\hline Brancos & $33,8 \%$ & $66,2 \%$ & & & & \\
\hline Negros & $28,7 \%$ & $71,3 \%$ & 1,018 & 0,716 & 0,927 & 1,117 \\
\hline Outros & $29,0 \%$ & $71,0 \%$ & 0,973 & 0,373 & 0,915 & 1,034 \\
\hline \multicolumn{7}{|l|}{ Idade (anos) } \\
\hline Entre 16 e 25 & $38,5 \%$ & $61,5 \%$ & & & & \\
\hline Entre 26 e 35 & $34,9 \%$ & $65,1 \%$ & 0,901 & 0,016 & 0,828 & 0,981 \\
\hline Entre 36 e 45 & $29,0 \%$ & $71,0 \%$ & 0,703 & $p<0,001$ & 0,643 & 0,769 \\
\hline Entre 46 e 55 & $27,1 \%$ & $72,9 \%$ & 0,626 & $p<0,001$ & 0,565 & 0,693 \\
\hline Entre 56 e 65 & $25,3 \%$ & $74,7 \%$ & 0,632 & $p<0,001$ & 0,559 & 0,714 \\
\hline 66 ou mais & $21,3 \%$ & $78,7 \%$ & 0,634 & $p<0,001$ & 0,529 & 0,760 \\
\hline
\end{tabular}


Tabela 2. (cont.)

\begin{tabular}{lcccccc}
\hline Escolaridade (anos de estudo) & \multicolumn{1}{l}{} \\
\hline Menos de 1 & $13,0 \%$ & $87,0 \%$ & & & & \\
1 a 5 & $18,3 \%$ & $81,7 \%$ & 1,437 & $p<0,001$ & 1,211 & 1,705 \\
6 a 9 & $25,4 \%$ & $74,6 \%$ & 1,850 & $p<0,001$ & 1,592 & 2,150 \\
10 a 12 & $34,4 \%$ & $65,6 \%$ & 2,527 & $p<0,001$ & 2,160 & 2,956 \\
13 a 14 & $45,5 \%$ & $54,5 \%$ & 3,719 & $p<0,001$ & 3,200 & 4,324 \\
15 ou mais & $51,3 \%$ & $48,7 \%$ & 5,719 & $p<0,001$ & 4,859 & 6,730 \\
Não determinados & $25,8 \%$ & $74,2 \%$ & 1,637 & 0,259 & 0,695 & 3,852 \\
\hline
\end{tabular}

Fonte: Elaboração com base nos dados da PNAD de 2015.

Por meio dos resultados da regressão logística, constata-se que as variáveis idade e escolaridade foram significativas, apresentando um $\mathrm{p}<0,05$. Desse modo, os resultados do modelo demonstram que quando o trabalhador estava na faixa etária entre 26 e 35 anos, havia redução de $9,9 \%$ da chance de praticar atividade física quando comparada à faixa entre 16 e 25 anos, adotada como categoria de referência para a análise de todas as faixas etárias. Além disso, para aqueles com idade acima de 66 anos, a chance de praticar a atividade era reduzida em 36,6\% (tabela 2).

A chance de praticar atividade física aumentava $271,9 \%$ quando o trabalhador possuía o nível de escolaridade de 12 a 14 anos de estudo, tomando-se como referência os que possuíam menos de um ano de estudo. Já para o grau de escolaridade de 15 anos ou mais, a chance aumentava para $471,9 \%$ (tabela 2 ).

Constatou-se que a maioria dos trabalhadores que viviam com companheiro ou cônjuge eram sedentários, bem como aqueles inseridos nas famílias compostas por casais com todos os filhos menores de 14 anos ou com 14 anos ou mais e por mães com todos os filhos menores de 14 anos. Do mesmo modo, a posse do domicílio resultou em percentuais similares na identificação dos trabalhadores fisicamente ativos. Esse fato também foi observado entre as regiões, sendo que os maiores e menores percentuais estão, respectivamente, no CentroOeste e no Norte (tabela 3).

Tabela 3. Aspectos do domicílio e da família segundo a prática de atividade física. Brasil, 2015 ( $n=82.019 .207)$

\begin{tabular}{|c|c|c|c|c|c|c|}
\hline Variável & $\begin{array}{r}\text { Praticou atividade } \\
\text { física }\end{array}$ & $\begin{array}{r}\text { Não praticou } \\
\text { atividade física }\end{array}$ & Odds Ratio & Valor $p$ & \multicolumn{2}{|c|}{$\begin{array}{r}\text { Intervalo de } \\
\text { Confiança }\end{array}$} \\
\hline & $n=25.564 .948$ & $n=56.454 .259$ & & & & \\
\hline \multicolumn{7}{|l|}{ Estado Civil } \\
\hline $\begin{array}{l}\text { Não vive com companheiro } \\
\text { ou cônjuge }\end{array}$ & $35,3 \%$ & $64,7 \%$ & & & & \\
\hline $\begin{array}{l}\text { Vive com companheiro ou } \\
\text { cônjuge }\end{array}$ & $28,8 \%$ & $71,2 \%$ & 0,836 & 0,002 & 0,747 & 0,934 \\
\hline \multicolumn{7}{|l|}{ Tipo de Família } \\
\hline Casal sem filhos & $31,0 \%$ & $69,0 \%$ & & & & \\
\hline
\end{tabular}


Tabela 3. (cont.)

\begin{tabular}{|c|c|c|c|c|c|c|}
\hline $\begin{array}{l}\text { Casal com todos os filhos } \\
\text { menores de } 14 \text { anos }\end{array}$ & $29,3 \%$ & $70,7 \%$ & 0,718 & $p<0,001$ & 0,658 & 0,785 \\
\hline $\begin{array}{l}\text { Casal com todos os filhos } \\
\text { com } 14 \text { anos ou mais }\end{array}$ & $32,5 \%$ & $67,5 \%$ & 0,932 & 0,162 & 0,844 & 1,029 \\
\hline $\begin{array}{l}\text { Casal com filhos menores } \\
\text { de } 14 \text { anos e com } 14 \text { anos } \\
\text { ou mais }\end{array}$ & $27,0 \%$ & $73,0 \%$ & 0,803 & 0,001 & 0,710 & 0,909 \\
\hline $\begin{array}{l}\text { Mãe com todos os filhos } \\
\text { menores de } 14 \text { anos }\end{array}$ & $27,2 \%$ & $72,8 \%$ & 0,569 & $p<0,001$ & 0,474 & 0,682 \\
\hline $\begin{array}{l}\text { Mãe com todos os filhos } \\
\text { com } 14 \text { anos ou mais }\end{array}$ & $34,0 \%$ & $66,0 \%$ & 0,879 & 0,091 & 0,758 & 1,021 \\
\hline $\begin{array}{l}\text { Mãe com filhos menores } \\
\text { de } 14 \text { anos e com } 14 \text { anos } \\
\text { ou mais }\end{array}$ & $25,0 \%$ & $75,0 \%$ & 0,673 & 0,001 & 0,534 & 0,848 \\
\hline Outros tipos de família & $34,5 \%$ & $65,5 \%$ & 1,011 & 0,881 & 0,880 & 1,160 \\
\hline \multicolumn{7}{|l|}{ Condição do Domicílio } \\
\hline Não próprio & $30,4 \%$ & $69,6 \%$ & & & & \\
\hline Próprio & $31,4 \%$ & $68,6 \%$ & 1,022 & 0,498 & 0,961 & 1,085 \\
\hline \multicolumn{7}{|l|}{ Região do Domicílio } \\
\hline Norte & $29,5 \%$ & $70,5 \%$ & & & & \\
\hline Nordeste & $31,0 \%$ & $69,0 \%$ & 1,222 & $p<0,001$ & 1,110 & 1,345 \\
\hline Sudeste & $30,8 \%$ & $69,2 \%$ & 0,999 & 0,983 & 0,905 & 1,102 \\
\hline Sul & $31,6 \%$ & $68,4 \%$ & 1,089 & 0,131 & 0,975 & 1,217 \\
\hline Centro-Oeste & $34,2 \%$ & $65,8 \%$ & 1,173 & 0,007 & 1,044 & 1,319 \\
\hline
\end{tabular}

Fonte: Elaboração com base nos dados da PNAD de 2015.

Os resultados do modelo logístico revelaram que a variável estado civil, algumas categorias da variável tipo de família e algumas categorias da variável região do domicílio foram significantes, com $\mathrm{p}<0,05$. Com isso, percebeu-se que, quando o trabalhador vivia com companheiro ou cônjuge, a razão de chance de praticar atividade física diminuía (16,4\%). De forma semelhante, a família composta por casal sem filhos e quaisquer outros tipos de família com significância menor que $5 \%$ apresentaram redução da chance de praticar exercícios físicos, com destaque para as trabalhadoras com todos os filhos menores de 14 anos, cuja diminuição foi de 43,1\% (tabela 3).
É notório que quando o domicílio do trabalhador se localizava na região Nordeste, a chance dessa prática aumentava $22,2 \%$ em relação aos que residiam na região Norte. A chance da região Norte foi $17,3 \%$ menor do que a da Centro-Oeste (tabela 3 ).

No que diz respeito às características do trabalho, os indivíduos mais ativos fisicamente trabalhavam de 15 a 39 horas por semana. Quanto à renda, a maioria dos que auferiam entre 12 e 15 salários mínimos praticava atividade física, sendo o mesmo constatado entre os militares. Além disso, o trabalhador em regime de trabalho formal foi mais ativo que o informal (tabela 4). 
Tabela 4. Prática de atividade física conforme características referentes às atividades laborais. Brasil, 2015 ( $n=82.019 .207)$

\begin{tabular}{|c|c|c|c|c|c|c|}
\hline Variável & $\begin{array}{r}\begin{array}{r}\text { Praticou atividade } \\
\text { física }\end{array} \\
n=25.564 .948\end{array}$ & $\begin{array}{r}\begin{array}{r}\text { Não praticou } \\
\text { atividade física }\end{array} \\
n=56.454 .259\end{array}$ & Odds Ratio & Valor $\mathrm{p}$ & \multicolumn{2}{|c|}{$\begin{array}{r}\text { Intervalo de } \\
\text { Confiança }\end{array}$} \\
\hline \multicolumn{7}{|l|}{ Horas Trabalhadas } \\
\hline Até 14 & $32,5 \%$ & $67,5 \%$ & & & & \\
\hline 15 а 39 & $33,3 \%$ & $66,7 \%$ & 0,846 & 0,007 & 0,749 & 0,954 \\
\hline 40 a 44 & $30,7 \%$ & $69,3 \%$ & 0,651 & $p<0,001$ & 0,578 & 0,733 \\
\hline 45 a 48 & $27,8 \%$ & $72,2 \%$ & 0,647 & $p<0,001$ & 0,563 & 0,743 \\
\hline 49 ou mais & $31,5 \%$ & $68,5 \%$ & 0,693 & $p<0,001$ & 0,606 & 0,793 \\
\hline \multicolumn{7}{|l|}{ Salário Mínimo* } \\
\hline Menos de 1 & $26,5 \%$ & $73,5 \%$ & & & & \\
\hline Entre 1 e 3 & $28,5 \%$ & $71,5 \%$ & 0,954 & 0,304 & 0,872 & 1,044 \\
\hline Entre 4 e 6 & $43,6 \%$ & $56,4 \%$ & 1,380 & $p<0,001$ & 1,218 & 1,564 \\
\hline Entre 7 e 9 & $51,7 \%$ & $48,3 \%$ & 1,767 & $p<0,001$ & 1,489 & 2,097 \\
\hline Entre 10 e 12 & $58,2 \%$ & $41,8 \%$ & 2,065 & $p<0,001$ & 1,541 & 2,767 \\
\hline Entre 13 e 15 & $59,7 \%$ & $40,3 \%$ & 2,365 & $p<0,001$ & 1,676 & 3,338 \\
\hline Acima de 15 & $37,9 \%$ & $62,1 \%$ & 1,229 & 0,031 & 1,020 & 1,482 \\
\hline \multicolumn{7}{|l|}{ Formalidade no Trabalho*^ } \\
\hline Trabalhadores informais & $27,9 \%$ & $72,1 \%$ & & & & \\
\hline Trabalhadores formais & $32,9 \%$ & $67,1 \%$ & 1,179 & 0,008 & 1,045 & 1,331 \\
\hline Não aplicável & $42,5 \%$ & $57,5 \%$ & & & & \\
\hline \multicolumn{7}{|l|}{ Posição na Ocupação } \\
\hline $\begin{array}{l}\text { Empregado com carteira de traba- } \\
\text { Iho assinada }\end{array}$ & $30,4 \%$ & $69,6 \%$ & & & & \\
\hline Militar & $79,3 \%$ & $20,7 \%$ & 5,586 & $p<0,001$ & 3,572 & 8,736 \\
\hline Funcionário público estatutário & $46,4 \%$ & $53,6 \%$ & 1,430 & $p<0,001$ & 1,289 & 1,587 \\
\hline $\begin{array}{l}\text { Outro empregado sem carteira de } \\
\text { trabalho assinada }\end{array}$ & $33,0 \%$ & $67,0 \%$ & 1,345 & $p<0,001$ & 1,171 & 1,546 \\
\hline $\begin{array}{l}\text { Trabalhador doméstico com cartei- } \\
\text { ra de trabalho assinada }\end{array}$ & $21,5 \%$ & $78,5 \%$ & 0,905 & 0,295 & 0,750 & 1,091 \\
\hline $\begin{array}{l}\text { Trabalhador doméstico sem cartei- } \\
\text { ra de trabalho assinada }\end{array}$ & $20,1 \%$ & $79,9 \%$ & 0,986 & 0,875 & 0,823 & 1,180 \\
\hline Trabalho por conta própria & $28,3 \%$ & $71,7 \%$ & 1,256 & $p<0,001$ & 1,129 & 1,399 \\
\hline Empregador & $42,5 \%$ & $57,5 \%$ & & & & \\
\hline $\begin{array}{l}\text { Trabalhador na produção para o } \\
\text { próprio consumo }\end{array}$ & $23,6 \%$ & $76,4 \%$ & 1,234 & 0,038 & 1,012 & 1,504 \\
\hline $\begin{array}{l}\text { Trabalhador na construção para o } \\
\text { próprio uso }\end{array}$ & $25,4 \%$ & $74,6 \%$ & 1,880 & 0,092 & 0,902 & 3,916 \\
\hline Não remunerado & $32,9 \%$ & $67,1 \%$ & 1,427 & 0,002 & 1,139 & 1,789 \\
\hline
\end{tabular}

Fonte: Elaboração com base nos dados da PNAD de 2015.

*O salário mínimo no ano da pesquisa era $\mathrm{R} \$ 788,00$

${ }^{* *}$ Foram considerados como formais os trabalhadores com carteira assinada, trabalhadores com contribuição previdenciária, servidores públicos civis e militares. 
As variáveis horas de trabalho e formalidade do trabalho, bem como algumas categorias das variáveis salário mínimo, e posições na ocupação, foram significativas, apresentando um $\mathrm{p}<0,05$ (tabela 4).

O modelo logístico demonstrou que quando se trabalhava de 15 a 39 horas semanais, a prática de atividade física se reduzia em $15,4 \%$ em relação aos que trabalhavam até 14 horas semanais. Da mesma forma, quando a carga horária era de 49 horas ou mais, a chance também se reduzia (30,7\%) (tabela 4).

A tabela 4 também permite concluir que a chance dessa prática aumentou 136,5\% para a faixa salarial entre 12 e 15 salários mínimos, tendo como referência os trabalhadores que auferiam menos de um salário mínimo. Essa chance aumentava outros $22,9 \%$ para rendas acima de 15 salários mínimos.

É importante acrescentar que no trabalho formal a chance é $17,9 \%$ maior do que no informal. Para finalizar, em relação à posição na ocupação, os que possuíam carteira de trabalho assinada, para qualquer outra posição na ocupação, mostraram um aumento da chance de praticar atividade física, ao nível de significância de 5\%. Cabe destacar que, quanto aos militares, a razão de chance aumentava em $458,6 \%$ (tabela 4 ).

\section{Discussão}

A prática de atividade física tornou-se uma ferramenta importante para a promoção da saúde e da qualidade de vida das pessoas, bem como para a redução das DCNT. O enfoque deste estudo foram as dificuldades encontradas pelo trabalhador para realizá-las, o que se justifica, em parte, pelas transformações ocorridas no contexto laboral. De fato, os resultados apontaram que a menor parte deles praticava atividade física, estando, portanto, suscetíveis a tais doenças, o que justifica discutir os fatores explicativos relevantes dessa ocorrência.

Nesta investigação, o número absoluto de homens que se exercitava foi superior ao de mulheres. Essa realidade pode ser explicada pelo fato de o total de homens da amostra ter sido maior que o de mulheres. No entanto, tal resultado equipara-se aos de outras investigações e justifica-se pelo fato de os homens usufruírem de maior tempo para tais atividades em momentos de lazer, geralmente por meio de algum esporte coletivo. Já as mulheres, deixam de se exercitar devido à dupla jornada de trabalho que enfrentam quase diariamente ${ }^{16,17}$.

Com relação à cor ou raça, evidências têm demonstrado que os adultos de cor branca se exercitam mais nos momentos de lazer ${ }^{\mathbf{1 8}}$. Em outro estudo, verificou-se que os participantes negros praticavam menos ginástica. Entretanto, apresentavam maior frequência da prática do futebol em relação aos que se declaravam brancos, fator compreensível, uma vez que esse esporte é incentivado como meio de ascensão social de classes majoritariamente compostas por negros ${ }^{19}$.

Nesse sentido, percebe-se a necessidade da implantação de políticas sociais voltadas à prática de atividade física pelos trabalhadores das diversas classes. Ressalta-se, ainda, a necessidade de inclusão das mulheres nessas ações, principalmente por seu menor tempo disponível que os homens.

Quanto à idade, notou-se nesta investigação que a prevalência de indivíduos que praticava atividade física se reduziu com o aumento da idade. A motivação para realizar exercícios físicos pode sofrer variações conforme a faixa etária. Na fase adulta, os indivíduos sentem-se mais motivados para tal, com o intuito de melhorar sua condição física, estética e socialização. Já para os idosos, a prática de atividade física inicia-se, prioritariamente, para manter e melhorar as condições de saúde e de qualidade de vida ${ }^{20}$.

As metas para o controle das DCNT no Brasil entre 2011 e 2022 contemplam o envelhecimento ativo do indivíduo como estratégia de promoção de saúde ${ }^{3}$. A isso, acresce-se o fato de a atividade física contribuir para a autonomia dessas pessoas na execução de suas tarefas, o que inclui a aptidão para o trabalho. 
Observou-se, ainda, que a prática de atividade física aumentou de acordo com o grau de escolaridade dos trabalhadores. Estudo realizado nas capitais no nordeste brasileiro evidenciou que os adultos que possuíam maior escolaridade tinham mais chances de exercitar-se, enquanto os de menor escolaridade apresentaram significativa redução das chances²1.

No que diz respeito à prática por grupos familiares, notou-se que os trabalhadores que viviam com companheiro ou cônjuge apresentavam menos chance de exercitar-se, bem como as mães com todos os filhos menores de 14 anos. A prática de atividade física é menor entre as mulheres casadas e entre as com filhos, dado o papel histórico que desempenham como cuidadoras da prole e responsáveis pelas tarefas domésticas. Além disso, muitas não têm com quem deixar os filhos para se exercitarem ${ }^{22}$.

Frente ao exposto, nota-se a necessidade de as empresas contribuírem com programas de atividade física no ambiente laboral. Essa iniciativa poderia integrar à prática de exercício os trabalhadores de baixa escolaridade, bem como as mulheres que possuem filhos, uma vez que é difícil a conciliação da tríade filhos, atividade física e trabalho. Desse modo, a prática de exercícios no ambiente laboral pode contribuir para o melhor desempenho, qualidade de vida e saúde dos trabalhadores.

Em relação à posse do domicílio, a participação do aluguel no orçamento familiar é maior entre as classes sociais de menor rendimento. Isso pode justificar o fato de os trabalhadores com casa própria apresentarem maiores chances de praticar atividade física ${ }^{23}$. O mesmo raciocínio pode ser aplicado à região de domicílio do trabalhador. Em uma investigação realizada com indivíduos de todo o Brasil, mostrou-se que as pessoas da região Nordeste são mais sedentárias do que as da região $\mathrm{Sul}^{24}$. Tal realidade pode ser explicada pelo fato de as condições socioeconômicas se deteriorarem à medida que se percorre o território nacional em direção ao Norte.

No que diz respeito às características do trabalho, notou-se que a prática de exercícios foi reduzida com o aumento da carga horária de trabalho. Esse achado pode ser justificado pelo tempo que o trabalhador despende executando suas atividades laborais. A 'falta de tempo', em razão do tempo direcionado ao trabalho, tem sido registrada na literatura como uma barreira para a prática de exercícios físicos ${ }^{25}$.

Quanto à renda, a relação entre os maiores níveis de renda e os maiores níveis de atividade física foi analisada em alguns estudos que reforçam o fato que pessoas com perfil socioeconômico desfavorecido, via de regra, residirem distantes do local de trabalho, trabalharem mais horas para complementar a renda, acarretando em menor tempo disponível para momentos de lazer e de prática de atividade física ${ }^{26}$. Além disso, aquelas com maior escolaridade e renda possuem maior possibilidade de acesso aos espaços para a prática, o que torna relevante o plano para o controle das DCNT, que prevê a ampliação dos espaços públicos para a realização de exercícios, democratizando o acesso ${ }^{3}$. Isso é importante, uma vez que parte dos trabalhadores sedentários alegaram problemas financeiros.

Diante disso, o fato de os trabalhadores formais serem fisicamente mais ativos do que os informais é explicado, possivelmente, em razão da maior escolaridade e renda dos primeiros. É notório que a escolaridade e o rendimento familiar dos trabalhadores informais são, em geral, baixos ${ }^{27}$.

Na variável posição na ocupação, verificou-se que o militar foi a única categoria que obteve um percentual de ativos superior ao de inativos, com maiores chances de prática de atividade física do que os trabalhadores com carteira assinada. Esse achado pode ser explicado pelo incentivo à prática de atividade física entre eles. A prática de exercícios melhora o desempenho dos militares no trabalho e sua função cognitiva, reduzindo o tempo de reação diante de alguma situação ${ }^{\mathbf{2 8}}$.

Entretanto, investigação realizada com 166 cadetes da Academia da Força Aérea Brasileira com idade entre 20 e 30 anos 
mostrou prevalência de fatores de risco cardiovascular entre eles, tais como hipertensão arterial sistêmica, excesso de peso, adiposidade elevada, hipercolesterolemia, alta ingestão de gordura saturada, mono e poli-insaturada e baixo consumo de fibras alimentares. São resultados preocupantes, uma vez que se esperava um perfil mais saudável e com menores prevalências desses fatores de risco entre jovens fisicamente ativos, o que revela riscos futuros para DCNT, especialmente os cardiovasculares ${ }^{29}$.

Frente aos fatores que estão associados à prática de atividade física de trabalhadores, pode-se considerar que ações de promoção da saúde são necessárias para essa população, as quais podem ser desenvolvidas pelas próprias empresas, tais como exercícios individuais diários, atividades de alongamento, incentivo à prática de atividades físicas coletivas no do ambiente laboral, dentre outras. Além disso, políticas governamentais tornam-se fundamentais para o incentivo das empresas e dos trabalhadores21. Com isso, espera-se que haja uma redução da inatividade física, dos números de DCNT e aumento na qualidade de vida e na capacidade de trabalhar desses indivíduos ${ }^{5,12}$.

Este estudo foi limitado pela disponibilidade de variáveis no banco de dados. Por esse motivo, não foi possível precisar a frequência da prática de atividade física recomendada pela OMS, isto é, de 150 minutos de atividade leve ou moderada ou 75 minutos de atividade intensa semanalmente. Apesar disso, a maior parte dos trabalhadores ativos praticava de quatro a sete vezes por semana de 40 a 60 minutos de exercícios.

$\mathrm{O}$ avanço do conhecimento proporcionado advém, principalmente, do importante nível de abrangência das informações nacionais analisadas.

\section{Conclusões}

Por meio das análises, foi possível avaliar os fatores associados à prática de atividade física de trabalhadores brasileiros. Com isso, conclui-se que o aumento da idade do trabalhador, a convivência com companheiro ou cônjuge, o fato de possuir filhos e o aumento da carga horária de trabalho são fatores associados à diminuição dessa prática. Por outro lado, os fatores que atuam em seu aumento são a elevação do grau de escolaridade, a região em que o trabalhador reside, o nível salarial mais elevado, a formalidade no trabalho e a posição que ocupa em seu trabalho.

Frente a isso, pode-se afirmar que os fatores que reduzem a prática de atividade física de os trabalhadores podem ser minimizados por meio do fortalecimento das políticas públicas. Ademais, os que diminuem o sedentarismo devem ser valorizados e estimulados por trabalhadores e gestores com o intuito de promover melhores condições de vida e trabalho.

\section{Colaboradores}

Silva AMR e Santos SVM contribuíram para a concepção e o planejamento da pesquisa, com a análise e a interpretação dos dados e com a aprovação da versão final do manuscrito. Lima CHF, Lima DJP e Robazzi MLC contribuíram para a elaboração do rascunho, na revisão crítica do conteúdo e na aprovação da versão final do manuscrito. 


\section{Referências}

1. World Health Organization. Global status report on noncommunicable diseases 2014 [internet]. Geneva: World Health Organization [acesso em 2018 out 10]. Disponível em: http://www.who.int/nmh/ publications/ncd-status-report-2014/en/.

2. Malta DC, França E, Abreu DMX, et al. Mortality due to noncommunicable diseases in Brazil, 1990 to 2015, according to estimates from the Global Burden of Disease study. Sao Paulo Med. J [internet]. 2017 maio-jun [acesso em 2018 out 10]; 135(3):213221. Disponível em: http://www.scielo.br/pdf/ spmj/v135n3/1806-9460-spmj-135-03-00213.pdf.

3. Malta DC, Andrade SSA, Santos MAS, et al. Tendências dos indicadores de atividade física em adultos: Conjunto de capitais do Brasil 2006-2013. Rev Bras Ativ Fís Saúde [internet]. 2015 [acesso em 2017 out 10]; 20(2):141-151. Disponível em: http:// rbafs.org.br/RBAFS/article/view/5059/4311.

4. Lee IM, Shiroma EJ, Lobelo F, et al. Effect of physical inactivity on major non communicable diseases worldwide: an analysis of burden of disease and life expectancy. Lancet [internet]. 2012 [acesso em 2017 out 22]; 380(9839):219-229. Disponível em: https://www.sciencedirect.com/science/ article/pii/S0140673612610319?via\%3Dihub.

5. World Health Organization. Global recommendations on physical activity for health [internet]. Geneva: World Health Organization. [acesso em 2017 out 22]. Disponível em: http://apps.who.int/iris/ bitstream/10665/44399/1/9789241599979_eng. pdf.

6. Caspersen CJ, Powell KE, Christenson GM. Physical activity, exercise, and physical fitness: definitions and distinction for health-relates research. Public Health Rep. 1985; 100(2):126-131.

7. Barbanti V. O que é esporte? Rev Bras Ativ Fís Saúde [internet]. 2006 [acesso em 2017 out 25]; 11(1):5458. Disponível em: http://rbafs.org.br/RBAFS/article/view/833/840.

8. Hallal PC, Andersen LB, Bull FC, et al. Global physical activity levels: surveillance progress, pitfalls and prospects. Lancet [internet]. 2012 [acesso em 2017 out 25]; 380(9839):20-30. Disponível em: https://www.sciencedirect.com/science/article/pii/ S0140673612606461.

9. Jesus GM, Jesus EFA. Nível de atividade física e barreiras percebidas para a prática de atividades físicas entre policiais militares. Rev Bras Ciênc Esport [internet]. 2012 [acesso em 2017 out 25]; 34(2):433-448. Disponível em: http://www.scielo.br/pdf/rbce/v34n2/a13v34n2.pdf.

10. Almeida GFP, Ribeiro MHA, Silva MACN, et al. Nascimento MDSB. Patologias osteomusculares como causa de aposentadoria por invalidez em servidores públicos do município de São Luís, Maranhão. Rev Bras Med Trab [internet]. 2016 [acesso em 2018 out 10]; 14(1):37-44. Disponível em: http://www.rbmt.org.br/details/21/pt-BR/patologias-osteomusculares-como-causa-de-aposentadoria-por-invalidez-em-servidores-publicos-do-municipio-de-sao-luis--maranhao.

11. Dejours C. A loucura do trabalho. São Paulo: Cortez; Oboré; 1992.

12. Brasil. Ministério da Saúde. Plano de ações estratégicas para enfrentamento das doenças crônicas não transmissíveis (DCNT) no Brasil 2011-2022. Brasília, DF: Ministério da Saúde. 2011.

13. Silva PLN, Pessoa DGC, Lila MF. Análise estatística de dados da PNAD: incorporando a estrutura do plano amostral. Ciênc. Saúde Colet. [internet]. 2002 [acesso em 2017 nov 2]; 7(4):659-670. Disponível em: http://dx.doi.org/10.1590/S1413- 
81232002000400005.

14. Instituto Brasileiro de Geografia e Estatística. Pesquisa Nacional por Amostra de Domicílios - 2015 [internet]. 2015 [acesso em 2017 nov 2]. Disponível em: https://ww2.ibge.gov.br/home/estatistica/populacao/trabalhoerendimento/pnad2015/ default.shtm.

15. Brasil. Fundação Nacional de Saúde. Resolução no 466, de 12 de dezembro de 2012. Pesquisas envolvendo seres humanos. Diário Oficial da União. 13 Dez 2012

16. Torquato ED, Gerage AM, Meurer ST, et al. Comparação do nível de atividade física medido por acelerômetro e questionário IPAQ em idosos. Rev Bras Ativ Fís Saúde [Internet]. 2016 [acesso em 2017 nov 10]; 21(2):144-153. Disponível: em: http:// rbafs.org.br/RBAFS/article/view/6438/5924.

17. Bauman AE, Reis RS, Sallis JF, et al. Correlates of physical activity: why are some people physically active and others not? Lancet [internet]. 2012 [acesso em 2017 nov 3]; 380(9839):258-271. Disponível em: https://doi.org/10.1016/S01406736(12)60735-1.

18. Del Duca GF, Nahas MV, Garcia LMT, et al. Prevalence and sociodemographic correlates of all domains of physical activity in Brazilian adults. Prevent. Med. [internet]. 2013 [acesso em 2017 nov 3]; 56(2):99-102. Disponível em: https://www.ncbi. nlm.nih.gov/pubmed/23200875.

19. Del Duca GF, Nahas MV, Hallal PC, et al. Leisure-Time Physical Activities among Adults in Florianopolis, State of Santa Catarina, Brazil: A Population-Based Study on the Characteristics of the Practices and the Practitioners. Ciênc. Saúde Colet. [internet]. 2014 [acesso em 2017 nov 5]; 9(11):4595-4604. Disponível em: http://dx.doi.or g/10.1590/1413812320141911.16732013.

20. Araújo C. Motivação e auto determinação para a prática de atividade física em idosos [dissertação] [internet]. Porto: Faculdade de Desporto da Universidade do Porto; 2015. 61 p. [acesso em 2018 out 10]. Disponível em: https://repositorioaberto.up.pt/bitstream/10216/80736/2/36737.pdf.

21. Tassitano RM, Mielke GI, Carvalho WRG, et al. Tendência temporal de indicadores da prática de atividade física e comportamento sedentário nas capitais da Região Nordeste do Brasil: 2006-2013. Rev Bras Ativ Fís Saúde [internet]. 2015 [acesso em 2018 out 10]; 20(2):152-164. Disponível em: http:// rbafs.org.br/RBAFS/article/view/5110/4312.

22. Nascimento TS, Mussi FC, Palmeira CS, et al. Fatores Relacionados à Inatividade Física no Lazer em Mulheres Obesas. Acta paul. Enferm. [internet]. 2017 [acesso em 2017 nov 5]; 30(3):308-315. Disponível em: http://dx.doi.org/10.1590/19820194201700047.

23. Kilsztajna S, Rossbach AC, Do Carmo MSN et al. Aluguel e rendimento domiciliar no Brasil. Rev Econ Contemp. 2009; 13(1):113-134.

24. Siqueira FV, Facchini LA, Piccini RX, et al. Atividade física em adultos e idosos residentes em áreas de abrangência de unidades básicas de saúde de municípios das regiões Sul e Nordeste do Brasil. Cad. Saúde Pública [internet]. 2008 [acesso em 2017 nov 3]; 24(1):39-54. Disponível em: http:// dx.doi.org/10.1590/S0102311X2008000100005.

25. Florindo AA, Hallal PC. Epidemiologia da Atividade Física. Ateneu; São Paulo: 2011.

26. Pitanga FG, Beck CC, Pitanga CPS, et al. Prevalência e fatores sociodemográficos e ambientais associados à atividade física no tempo livre e no deslocamento em adultos. Motri [internet]. 2014 [acesso em 2017 nov 3]; 10(1):03-13. Disponível em: http://dx.doi.org/10.6063/motricidade.10(1).1246.

27. Pamplona JB. Mercado de trabalho, informalidade e comércio ambulante em São Paulo. Rev. Bras. Est Pop [internet]. 2013 [acesso em 2017 nov 
3]; 30(1):225-249. Disponível em: http://dx.doi. org/10.1590/S0102-30982013000100011.

28. Dominsky FH, Matias TS, Cardoso TE, et al. Motivação para a Prática de Exercícios está Relacionada ao Tempo de Reação dos Policiais Civis de Unidades de Operações Especiais. Rev Cub Med Militar. 2015; 44(2):195-206.

29. Hilgenberg FE, Santos ASAC, Silveira EA, et al. Fa- tores de risco cardiovascular e consumo alimentar em cadetes da Academia da Força Aérea Brasileira. Ciênc. Saúde Colet. [internet]. 2016 [acesso em 2017 nov 3]; 21(4):1165-1174. Disponível em: https://doi. org/10.1590/1413-81232015214.15432015.

Recebido em 24/07/2018

Aprovado em 25/11/2018

Conflito de interesses: inexistente

Suporte financeiro: não houve 